\title{
Redes sociales como estrategia académica en la educación superior: ventajas y desventajas
}

\section{Sonia Janneth Limas Suárez}

https://orcid.org/oooo-0002-1145-3713 Universidad Pedagógica y Tecnológica de Colombia

sonia.limas@uptc.edu.co

\section{Gloria Vargas Soracá}

https://orcid.org/0000-0003-0598-7492 Universidad Pedagógica y Tecnológica de Colombia

gloria.vargaso2@uptc.edu.co

\section{Resumen}

Las redes sociales aplicadas en la educación superior se han convertido en una herramienta innovadora. Por eso es relevante determinar sus ventajas y desventajas como estrategia académica. Este estudio utiliza el método descriptivo, empírico y cuantitativo en el análisis de una muestra de 191 estudiantes de un programa académico profesional en una universidad oficial colombiana. Los resultados muestran que las redes sociales se deben incorporar en la educación superior como política institucional y no solo como eventos episódicos o coyunturales. Esto exige un proceso educativo paralelo orientado a forjar en los estudiantes la responsabilidad y la autonomía en el uso de este recurso.

\section{Palabras claves (Fuente: tesauro de la Unesco)}

Enseñanza superior; innovación educacional; métodos de enseñanza; pedagogía universitaria; TIC en la educación.

Recepción: 18/07/2020 | Envío a pares: 16/11/2020 | Aceptación por pares: 8/12/2020 | Aprobación: 9/12/2020 


\title{
Social Media as an Academic Strategy in Higher Education: Pros and Cons
}

\begin{abstract}
Social media applied to higher education have become a groundbreaking tool. Then, it is vital to determine its advantages and disadvantages as an academic strategy. This study employs a descriptive, empirical, quantitative method in analyzing a sample of 191 students from an undergraduate program at a Colombian public university. The results suggest that social media should be incorporated into higher education as an institutional policy and not only episodically or circumstantially, requiring a parallel educational process to foster students' responsibility and autonomy in using this resource.
\end{abstract}

\section{Keyword (Source: Unesco Thesaurus)}

Higher education; educational innovations; teaching methods; university pedagogy; ICT in education. 


\title{
Redes sociais como estratégia acadêmica no ensino superior: vantagens e desvantagens
}

\begin{abstract}
Resumo
As redes sociais aplicadas no ensino superior têm se tornado uma ferramenta inovadora. Por isso, é relevante determinar suas vantagens e desvantagens como estratégia acadêmica. Neste estudo, é utilizado o método descritivo, empírico e quantitativo na análise de uma amostra de 191 estudantes de um programa acadêmico profissional em uma universidade oficial colombiana. Os resultados indicam que as redes sociais devem ser incorporadas no ensino superior como politica institucional e não somente como eventos episódicos ou conjunturais. Isso exige um processo educativo paralelo orientado a forjar nos estudantes a responsabilidade e a autonomia no uso desse recurso.
\end{abstract}

Palavras-chave (Fonte: tesauro da Unesco)

Ensino superior; inovação educacional; métodos de ensino; pedagogia universitária; TIC na educação. 
Las redes sociales, surgidas del desarrollo tecnológico, han permeado el escenario educativo mediante la incursión de tecnologías y herramientas que enriquecen y fortalecen los procesos de formación académica. De este modo emergen como innovaciones útiles para la educación superior (Chacón y Limas, 2019), resultado de la revolución tecnológica propia de la era digital en el siglo XXI.

Como estrategia en los procesos de formación académica, las redes sociales se han convertido en un medio de interacción entre los docentes y los estudiantes, por medio de la utilización de diferentes aplicaciones que facilitan el desarrollo de los procesos educativos. En términos conceptuales, Prato y Villoria (2010) las definen como sistemas que permiten establecer relaciones con otros usuarios y espacios de intercambio de información y generación de relaciones e interacción entre distintas personas. Por su lado, Valenzuela (2013) denomina red social no solo la agrupación de personas que la componen, sino el sistema de alojamiento y ofrecimiento del servicio, y sostiene, además, que son requeridas para generar vínculos entre los usuarios sin que esto implique necesariamente un interés común. Por último, Cabero et al. (2016) afirman que las redes sociales se han constituido en grandes potenciales tecnológicos, pues ofrecen una gran variedad de servicios y ventajas para sus usuarios, entre ellos, la comunicación fácil, con lo cual derriban fronteras y dan acceso a una comunidad global. Estas definiciones se centran en la comunicación como factor elemental a partir de la interrelación con estas herramientas.

En la esfera educativa, Cabero et al. (2016) afirman que las redes sociales se han venido incorporado a la educación como herramientas que avanzan hacia entornos comunicacionales de aprendizaje y colaboración, cuya aplicación ha venido creciendo de manera exponencial. Por su parte, Marín y Cabero (2019) señalan que son instrumentos básicos utilizados en la sociedad del conocimiento y resaltan su crecimiento exponencial en los entornos de formación, al punto que no solo los centros educativos las incorporan para tareas administrativas y de información a las familias, sino también los docentes, como herramientas e instrumentos para transmitir información y crear entornos de trabajo colaborativos. Estas definiciones resaltan el uso de las redes sociales en la práctica educativa, como lazo comunicacional entre la comunidad educativa, lo que además favorece el desarrollo de otras metodologías y ambientes de aprendizaje.

De igual modo, diversos estudios (Chacón y Limas, 2019; Marín y Cabero, 2019; Cantillo y Moreno, 2016; Cornejo y Parra, 2016; Chávez, 2014) coinciden en afirmar que las redes sociales toman cada día más fuerza y presencia en las universidades, lo que induce cambios en los modelos educativos y en el acceso y las formas de aprendizaje y las convierte en un aliado innovador que conlleva "la mejora en el proceso enseñanza-aprendizaje y en la calidad de la educación" (Cornejo y Parra, 2016).

A nivel mundial, las redes sociales más usadas son Facebook, YouTube y Whatsapp, según el reporte anual Global Digital Report (We Are Social, 2019). En América Latina predomina Facebook, Whatsapp, YouTube y Twitter, de acuerdo con el informe Digital News Report (Newman et al., 2019). Localmente, en México, Argentina y Colombia priman Facebook, Whatsapp y YouTube, según Global Digital Report y Digital News Report, y en España optan por Whatsapp, Facebook y YouTube, de acuerdo con el Estudio Anual Redes Sociales (IAB Spain, 2019) y el reporte Global Digital Report.

No obstante, un problema emerge cuando, a pesar de que existen estudios sobre la incursión de las redes sociales en la educación, no se evidencian las ventajas y desventajas que traen para la educación superior, tomando como referente el análisis de estudios primarios y secundarios. Dada esta situación, importa saber cuáles son las ventajas y desventajas de las redes sociales como estrategia académica en la educación superior. A partir de esta pregunta, y teniendo en cuenta el notorio uso de las 
redes sociales en universidades de América Latina, España y México (Islas y Carranza, 2011; Castillo, 2013), el presente artículo tiene como propósito establecer las ventajas y desventajas de las redes sociales como estrategia académica en la educación superior. La implementación de estas herramientas permite avanzar y apostar por una educación competitiva, sin olvidar que ello tiene connotaciones de distinta indole: desde la favorabilidad (Cabrero y Marín, 2014; Muñoz et al., 2013; Islas y Carranza, 2011; Sáenz, 2010) hasta un determinado pesimismo que las cuestiona (Marín y Cabero, 2019; Martínez y Ramírez, 2016), razones adicionales que ameritan el desarrollo de la presente investigación.

\section{Métodología}

El presente estudio busca establecer las ventajas y desventajas de las redes sociales como estrategia académica en la educación superior a partir del estudio realizado con estudiantes del programa de Administración Comercial y Financiera de la Universidad Pedagógica y Tecnológica de Colombia. El método usado fue descriptivo, empírico y cuantitativo.

La población del estudio está compuesta por estudiantes universitarios de Administración Comercial y Financiera que cursan de 1 hasta 10 semestre, en la sede principal del campus universitario en Tunja (Boyacá, Colombia) y los demás Centros Regionales de Educación a Distancia (Cread) donde funciona el programa (Tabla 1), matriculados para el primer periodo académico 2019. Se usó el método de muestreo probabilístico, de tipo aleatorio simple, con 191 estudiantes, de los cuales $81 \%$ son mujeres y el $19 \%$ son hombres, con edades que oscilan entre los 18 a 60 años; en lo ocupacional, el 56\% de los estudiantes laboran y el $44 \%$ solamente estudian. Se tuvo un margen de error de 0,05\%para un nivel de confianza del 95\%.

El instrumento utilizado para la recopilación de la información fue la encuesta, organizada para establecer las ventajas y desventajas de las redes so- ciales en el proceso de formación académica en el programa Administración Comercial y Financiera a partir de la percepción de los estudiantes. Con este fin, se construyó un cuestionario estructurado con cinco preguntas para calificar 24 ítems mediante preguntas cerradas, para distinguir con claridad la experiencia del encuestado. En la validación del instrumento, se trabajó en la revisión documental, con el propósito de facilitar la identificación de las variables más pertinentes al objetivo del estudio. Igualmente, se consultaron estudios con similares instrumentos validados, para posterior contrastación con el juicio de expertos. Además, se hizo uso de la matriz de compilación de información, a fin de contar con un instrumento adecuado al objetivo trazado. Para determinar la confiabilidad del instrumento, se tomó una muestra piloto de 57 estudiantes y se aplicó el método Kuder-Richardson 20 (KR-20), que arrojó como resultado o,81, lo que determina que el instrumento es altamente confiable.

Tabla 1. Datos de la muestra por sede y Cread

\begin{tabular}{|l|c|c|}
\hline \multicolumn{1}{|c|}{ Sede/Cread } & Muestra (N) & Porcentaje \\
\hline Tunja & 132 & $69 \%$ \\
\hline Duitama & 27 & $14 \%$ \\
\hline Bogotá & 13 & $7 \%$ \\
\hline Garagoa & 11 & $6 \%$ \\
\hline Yopal & 6 & $3 \%$ \\
\hline Rondón & 2 & $1 \%$ \\
\hline TOTAL & 191 & $100 \%$ \\
\hline
\end{tabular}

Fuente: elaboración propia.

Para el procesamiento y organización de los resultados, se trabajó con base en la matriz de compilación de información y el programa informático de Excel. Se compiló la información y clasificó por variable de análisis: conocimiento, razones de utilización, redes de mayor utilización, ventaja y desventaja, y luego se establecieron los ítems para cada variable y sus respuestas. Este método de codificación permite estructurar las fuentes de información primaria de una manera más sistemática, determinar categorías y jerarquías dentro del proceso de sistematización e integrar da- 
tos que en algún momento pueden ser relevantes para el posterior curso de investigación, en la etapa de planteamiento de propuestas.

La matriz de compilación y organización de la información codificada contempló cuatro columnas. En la primera, titulada "Variable de análisis", se relaciona cada variable del estudio, el conocimiento, las razones de utilización, las redes de mayor utilización y su ventaja y desventaja. En la segunda columna, de "Ítems", se los clasificó por cada variable de estudio. La tercera columna, sobre "Frecuencia absoluta (n1)", corresponde al total de respuestas obtenidas por cada item. Por último, la cuarta columna, de "Frecuencia relativa (f1)", corresponde al porcentaje de respuestas obtenido por cada item.

Para analizar la información se trabajó con el paquete estadístico de Excel, mediante la observación de datos resultado de tablas que muestran el comportamiento de cada ítem y variable de estudio.

\section{Resultados}

En el análisis para establecer las ventajas y desventajas de las redes sociales como estrategia académica en la educación superior, a partir del estudio realizado con estudiantes del programa de Administración Comercial y Financiera de la Universidad Pedagógica y Tecnológica de Colombia, los resultados que se presentan se obtuvieron siguiendo el modelo de estudio descriptivo, empírico y cuantitativo y demuestran que las redes sociales despiertan en los estudiantes un marcado interés. En tal sentido, cuando se indaga en el estudiantado por el conocimiento de las redes sociales, se puede observar que el 100\% sílas conocen (Tabla 2).

Tabla 2. Conocimiento de las redes sociales en el estudiantado

\begin{tabular}{|c|c|c|}
\hline Categoria (Xi) & $\begin{array}{c}\text { Frecuencia } \\
\text { absoluta (ni) }\end{array}$ & $\begin{array}{c}\text { Frecuencia relativa } \\
\text { (fi) }\end{array}$ \\
\hline Sí & 191 & $100 \%$ \\
\hline No & 0 & $0 \%$ \\
\hline TOTAL & 191 & $100 \%$ \\
\hline
\end{tabular}

Fuente: elaboración propia.
Igualmente, se encuentra que los estudiantes utilizan las redes sociales para el desarrollo de los procesos de formación académica, por iniciativa propia y de los docentes, y a pesar de que el programa no cuenta con una política institucional de uso y manejo de redes sociales en el proceso de formación, los estudiantes encuentran en esta herramienta un importante apoyo no solo como fuente de comunicación, sino también para el desarrollo de los procesos educativos. Así, el 95\% del estudiantado utiliza las redes sociales para comunicarse, el $75 \%$ las emplea como herramienta de estudio y el $49 \%$ las destina como medio de entretenimiento y ocio (Tabla 3).

Tabla 3. Razones de utilización de las redes sociales en el estudiantado

\begin{tabular}{|l|c|c|}
\hline \multicolumn{1}{|c|}{ Categoria (Xi) } & $\begin{array}{c}\text { Frecuencia } \\
\text { absoluta (ni) }\end{array}$ & $\begin{array}{c}\text { Frecuencia } \\
\text { relativa (fi) }\end{array}$ \\
\hline Comunicarse & 181 & $95 \%$ \\
\hline $\begin{array}{l}\text { Herramienta de } \\
\text { estudio }\end{array}$ & 143 & $75 \%$ \\
\hline $\begin{array}{l}\text { Entretenimiento } \\
\text { yocio }\end{array}$ & 94 & $49 \%$ \\
\hline
\end{tabular}

Fuente: elaboración propia.

En cuanto a las redes sociales de mayor utilización para el desarrollo de los procesos de formación académica de la Universidad, se puede observar una marcada preferencia en el estudiantado por Whatsapp, herramienta que les facilita enviar y recibir información en el entorno educativo, particularmente para cada una de las asignaturas. A esta le sigue YouTube, medio audiovisual con el cual los estudiantes afianzan su proceso de aprendizaje, al facilitarles la conceptualización y socialización de nuevo conocimiento y ser un canal de aprendizaje más interactivo, especialmente en estudiantes en los que predomina la percepción visual. La siguiente red social es Facebook, herramienta que favorece la observación de información a nivel general, empresarial y específico del campo de formación. Por último, quedan Instagram, aplicación para compartir fotos y videos de apoyo en el campo académico, y Twiter, que faci- 
lita el dialogo, el debate y la discusión, así como la investigación y notificación de anuncios y mensajes breves. En consecuencia, el 95\% de los estudiantes utilizan Whatsapp, el 74\% acceden a YouTube, el 21\% usan Facebook, y 9\% y 4\% emplean Instagram y Twiter, respectivamente, para el desarrollo de los procesos de formación académica universitaria (Tabla 4).

Tabla 4. Redes sociales de mayor utilización por los estudiantes

\begin{tabular}{|c|c|c|}
\hline Categoria (Xi) & $\begin{array}{c}\text { Frecuencia } \\
\text { absoluta (ni) }\end{array}$ & $\begin{array}{c}\text { Frecuencia } \\
\text { relativa (fi) }\end{array}$ \\
\hline WhatsApp & 181 & $95 \%$ \\
\hline YouTube & 141 & $74 \%$ \\
\hline Facebook & 40 & $21 \%$ \\
\hline Instagram & 17 & $9 \%$ \\
\hline Twiter & 8 & $4 \%$ \\
\hline
\end{tabular}

Fuente: elaboración propia.

Por consiguiente, en la utilización de las redes sociales los estudiantes encuentran un aliado creativo e innovador para los procesos de formación, el cual, además de facilitarles la comunicación, también los ayuda en su proceso de enseñanza-aprendizaje, por tratarse de herramientas dinamizadoras que se pueden introducir en el ámbito educativo a fin de fortalecer y enriquecer sus procesos. En este sentido, las ventajas encontradas fueron:

1. Facilitan el diálogo y la comunicación entre compañeros (82\%).

2. Facilitan la comunicación con el docente (75\%).

3. Incrementan la colaboración entre compañeros en el desarrollo de actividades (61\%).

4. Ayudan al aprendizaje de temas específicos (56\%).

5. Ayudan en la interacción y el debate sobre temas del curso (53\%).

6. Se publica y se comparte recursos y/o contenidos afines a los temas del curso (47\%).

7. Facilitan la investigación en los temas y, por tanto, la elaboración de actividades del curso (47\%).
8. Proporcionan contenidos e información valiosa para la elaboración de actividades del curso (18\%).

9. Dan acceso a cualquier hora (2\%).

Comparando los resultados, en la Tabla 5 se observa que las ventajas 1 y 2 ofrecen los porcentajes de mayor representación, al facilitar la comunicación entre compañeros (82\%) y con los docentes (75\%), lo que significa que las redes sociales predominan como canal de comunicación en los procesos educativos. Sin embargo, hay mayores ventajas en las categorías 3 a 9, donde las redes sociales apor$\tan$ al proceso de enseñanza-aprendizaje, con porcentajes de respuesta entre el $61 \%$ y el $10 \%$, para un número de 7 ventajas, que giran en torno a este propósito. En orden descendente, los puntajes mayores se encuentran en la ventaja 3 , que incrementa la colaboración entre compañeros en desarrollo de las actividades del curso (61\%), seguida de las ventajas 4 y 5 , sobre ayuda en el aprendizaje de temas específi$\cos (56 \%)$ y en la interacción y el debate sobre temas del curso (53\%); por último, quedan las ventajas $6 \mathrm{y}$ 7 , consistentes, respectivamente, en que en las redes sociales se publica y se comparte recursos y/o contenidos afines a los temas del curso (47\%), y ellas facilitan la investigación en los temas y, por tanto, en la elaboración de las actividades del curso (47\%). Estos resultados indican que las redes sociales también son percibidas como herramientas que facilitan, fortalecen y enriquecen los procesos educativos.

Los puntajes menores se encuentran en las ventajas 8 y 9 , relativas a la obtención de contenidos e información valiosa para la elaboración de las actividades del curso (18\%) y al acceso a cualquier hora (10\%). Esta menor ventaja es percibida así por los estudiantes debido a que las redes sociales no están ligadas como herramientas de apoyo y acompañamiento al desarrollo de los cursos y sus actividades, sino que su uso es iniciativa del estudiantado en sus actividades académicas; por consiguiente, las búsquedas en algunos casos no son tan efectivas a los propósitos del curso. Para contrarrestar esto, se requiere incorporarlas a los procesos educativos, a fin 
de conducir la efectiva utilización de las mismas en el desarrollo de los cursos en conjunto con el cuerpo docente, de manera que la contribución sea mayor a los propósitos de clase.

De acuerdo con los resultados, el estudio conlleva valorar el aporte de las redes sociales en los procesos de formación, dado que los estudiantes encuentran en ellas un recurso valioso y significativo para su formación, de tal manera que se pueden implementar en los programas académicos como política institucional, por las ventajas que estas herramientas generan y representan en los procesos educativos.

Tabla 5. Ventajas de las redes sociales en la formación académica

\begin{tabular}{|c|c|c|}
\hline Categoria (Xi) & 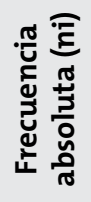 & 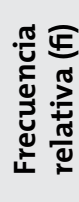 \\
\hline $\begin{array}{l}\text { 1. Facilitan el diálogo y la comunicación } \\
\text { entre compañeros }\end{array}$ & 157 & $82 \%$ \\
\hline $\begin{array}{l}\text { 2. Facilitan la comunicación con el } \\
\text { docente }\end{array}$ & 143 & $75 \%$ \\
\hline $\begin{array}{l}\text { 3. Incrementan la colaboración entre } \\
\text { compañeros en el desarrollo de } \\
\text { actividades }\end{array}$ & 117 & $61 \%$ \\
\hline $\begin{array}{l}\text { 4. Ayudan en el aprendizaje de temas } \\
\text { específicos }\end{array}$ & 107 & $56 \%$ \\
\hline $\begin{array}{l}\text { 5. Ayudan en la interacción y el debate } \\
\text { sobre temas del curso }\end{array}$ & 101 & $53 \%$ \\
\hline $\begin{array}{l}\text { 6. Se publica y se comparte recursos } \\
\text { y/o contenidos afines a los temas del } \\
\text { curso }\end{array}$ & 90 & $47 \%$ \\
\hline $\begin{array}{l}\text { 7. Facilitan la investigación en los temas } \\
\text { y, por tanto, la elaboración de las } \\
\text { actividades del curso }\end{array}$ & 90 & $47 \%$ \\
\hline $\begin{array}{l}\text { 8. Proporcionan contenidos e informa- } \\
\text { ción valiosa para la elaboración de las } \\
\text { actividades del curso }\end{array}$ & 34 & $18 \%$ \\
\hline 9. Dan acceso a cualquier hora & 19 & $10 \%$ \\
\hline
\end{tabular}

Fuente: elaboración propia.
Por otra parte, el estudio estableció igualmente las siguientes desventajas de las redes sociales en los procesos de formación:

1. Dependencia de las redes sociales, que desborda tiempos y límites (60\%).

2. Distracción exagerada y exceso de información (51\%).

3. Reducción del contacto físico entre compañeros y con los docentes (49\%).

4. Problemas de veracidad y confiabilidad de la información consultada (38\%).

5. Dificultades de acceso a internet (21\%).

6. Mala administración del tiempo y desplazamiento de otras prioridades (2\%).

En el análisis de los resultados, la Tabla 6 muestra que los porcentajes de mayor representación de respuesta están en las desventajas 1 y 2, consistentes en la dependencia de las redes sociales, que desborda tiempos y límites (60\%), y en la distracción exagerada y el exceso información (51\%), respectivamente, desventajas que vienen del mal uso de las redes sociales, en cuanto a tiempos, límites y alcances de la información. Respecto de las desventajas 3 y 4 , relativas a la reducción del contacto físico entre compañeros y docentes (49\%) y a los problemas con la veracidad y confiabilidad de la información consultada (38\%), respectivamente, los estudiantes que ingresan a un programa universitario virtual con encuentros presenciales han de vérselas con una modalidad de estudio nueva para ellos, pues su formación anterior se impartía en modalidad presencial. La nueva modalidad de estudio les exige, por tanto, mayor utilización de las redes sociales, cuando no se acude solamente a los medios que dispone el curso, ya sea como medio de comunicación y/o como apoyo en las actividades académicas, todo lo cual explica las respuestas obtenidas. Con relación a la desventaja 4, es decir, a los problemas de veracidad y confiabilidad de la información consultada, 
ella es consecuencia de no hacer un correcto direccionamiento en las búsquedas a través de las redes sociales, además que su utilización es iniciativa de los estudiantes y no está articulada con los cursos del programa.

Tabla 6. Desventajas de las redes sociales en la formación académica

\begin{tabular}{|c|c|c|}
\hline Categoria (Xi) & 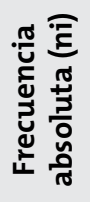 & 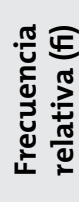 \\
\hline $\begin{array}{l}\text { 1. Dependencia de las redes sociales, } \\
\text { que desborda tiempos y límites }\end{array}$ & 115 & $60 \%$ \\
\hline $\begin{array}{l}\text { 2. Distracción exagerada y exceso de } \\
\text { información }\end{array}$ & 98 & $51 \%$ \\
\hline $\begin{array}{l}\text { 3. Reducción del contacto físico entre } \\
\text { compañeros y con los docentes }\end{array}$ & 94 & $49 \%$ \\
\hline $\begin{array}{l}\text { 4. Problemas de veracidad y } \\
\text { confiabilidad de la información } \\
\text { consultada }\end{array}$ & 72 & $38 \%$ \\
\hline 5. Dificultades de acceso a internet & 41 & $21 \%$ \\
\hline $\begin{array}{l}\text { 6. Mala administración del tiempo y } \\
\text { desplazamiento de otras prioridades }\end{array}$ & 2 & $1 \%$ \\
\hline
\end{tabular}

Fuente: elaboración propia.

Finalmente, los porcentajes de respuesta más bajos se ubican en las desventajas 5 y 6 , acerca de las dificultades de acceso a internet (21\%) y la mala administración del tiempo y el desplazamiento de otras prioridades (1\%). Acá se observa que para acceder a las redes sociales se necesita acceso a internet, ya sea desde los dispositivos móviles o a través de los equipos de cómputo; de lo contrario, se limita su uso. Adicionalmente, hay zonas en el país en las que no se cuenta con señal o cubrimiento de internet, lo que dificulta el acceso a las redes sociales, o donde se requiere comprar el servicio, a lo que se suma que son limitadas las áreas geográficas con conectividad subsidiada, aparte de los campus universitarios, donde en ocasiones no se cuenta con conexión wifi gratuita. La desventaja 6, al igual que la desventaja 1, vienen más del mal uso de las redes sociales en cuanto a los tiempos que se les dedican. En conse- cuencia, es necesario establecer pautas de buen uso de las redes sociales en lo que concierne al tiempo, el acceso y los límites de los estudiantes, para lo cual es importante el acompañamiento y asesoría de los docentes y su rol en el proceso, así como la capacitación a los estudiantes sobre la utilización y buenas prácticas en las redes sociales. Para contrarrestar a nivel institucional las desventajas expuestas, es necesario establecer una normativa de uso y manejo de las redes sociales en el contexto educativo.

\section{Discusión y conclusiones}

La era digital ha representado verdaderos retos y transformaciones en la educación superior como resultado de la revolución tecnológica y ha permeado los procesos educativos modernizando el sector (Limas, 2019). En tal sentido, la incursión de las redes sociales en el campo educativo ya es objeto de estudio tanto en universidades de América Latina como en el resto del mundo, por ser consideradas un aliado innovador en la educación superior.

En estudios previos se considera que las redes sociales "pueden ser utilizadas como ventaja competitiva para trabajar en forma colaborativa, ya que son plataformas gratuitas y accesibles, que favorecen la motivación y el interés de los estudiantes en el momento de buscar estrategias que beneficien en su aprendizaje" (Islas y Carranza, 2011); además, abren un abanico de posibilidades para el trabajo académico con los estudiantes $y$, a su vez, se han convertido en una de las formas de interacción más usadas por el estudiantado (Limas y Jaimes, 2017; Merchán et al., 2018).

En consecuencia, sobre las ventajas y desventajas de las redes sociales como estrategia académica en la educación superior, los resultados de este estudio indican que los estudiantes encuentran en las redes sociales un aporte valioso, significativo e innovador en su proceso educativo, por lo que pueden ser consideradas un factor de innovación en la educación superior (Islas y Carranza, 2011; Merchán et al., 2018). 
De igual forma, los estudiantes perciben en las redes sociales un aliado para el desarrollo de los procesos académicos. Así, las ventajas encontradas en este estudio no solo consisten en facilitar la comunicación entre compañeros y docentes, interactuando como canal de comunicación en los procesos educativos (Castillo, 2013), sino, además, en ser percibidas como herramientas que facilitan, fortalecen y enriquecen los procesos de formación académica en la educación superior.

Desde esta perspectiva, se hallan estudios en línea con los resultados, donde las redes sociales son consideradas una fuente de aprendizaje, ya que sus herramientas potencian las actividades de enseñanza-aprendizaje (Brito et al., 2012; Merchán et al., 2018). A la vez, 1) ellas fomentan un nuevo modelo de aprendizaje, que se caracteriza por ser sumamente interactivo, creativo y participativo para beneficio del estudiantado y del cuerpo docente (Redem, 2017; Islas y Carranza, 2011; Shang et al., 2011); 2) favorecen la creación de mejores espacios educativos y con calidad, en entornos digitales basados en las tecnologías de la información y la comunicación (TIC) (Colina, 2008; García et al., 2009; Brenes, 2010); y c) juegan un papel fundamental de transformación de las prácticas de enseñanza-aprendizaje, al convertirse en una realidad y una exigencia en los procesos académicos, a los que les plantean retos (Ángel y Patiño, 2018).

Otros estudios consideran que, como el proceso de enseñanza-aprendizaje en la educación superior requiere organizarse de varias formas, en las clases se puede optar por una conferencia, un seminario, una práctica, un encuentro, etc. (Hernández e Infante, 2017), espacios donde las redes sociales se convierten en un medio que favorece el aprendizaje, al compartir conocimientos, desarrollar habilidades, destrezas y valores, todo lo cual redunda en la formación integral de los estudiantes. Estas tecnologías digitales traen entonces importantes oportunidades asociadas a los procesos académicos; de ahí el valor de integrarlas en el contexto educativo, obviamente de acuerdo con el contexto y tipo de actividad académica a desarrollar (López, 2017).

Además, se constata que la implementación de las redes sociales en las actividades académicas apoya la enseñanza (Rojas, 2016), juega un papel protagónico en los distintos ambientes académicos y de formación y contribuye al mejoramiento de la calidad educativa como resultado de la incursión de las TIC en la educación superior (Ángel y Patiño, 2018; Escorcia y Jaimes, 2015; Martínez, 2014).

De esta manera, entre los resultados del estudio, las anteriores ventajas de las redes sociales en el campo universitario están en concordancia con el aporte de la Universidad Católica de Valencia (Florido, 2016):

1. Para el diálogo y la comunicación, sin duda las redes sociales permiten crear vínculos con los compañeros y promover el contacto para intercambiar y compartir información y experiencias, al tiempo que estimulan el desarrollo de competencias comunicativas orientadas al análisis de la información, el uso de las TIC, el trabajo en equipo y las habilidades multitarea (Silva et al., 2012).

2. En la comunicación con el docente, ayudan durante la asesoría, la resolución de dudas y el acompañamiento en los procesos académicos; a su vez, posibilitan el monitoreo progresivo del estudiante, lo que estimula un mayor contacto y proximidad entre los estudiantes y los docentes mediante una comunicación fluida y dinámica (Romero, 2012; Laguna, 2013; Merchán et al., 2018), más allá de los horarios habituales en el desarrollo de las actividades académicas (Educaweb, 2018).

3. Respecto de la colaboración entre compañeros para el desarrollo de las actividades, las redes sociales les permiten compartir información de los cursos, los motivan a participar en el desarrollo de las actividades e incluso potencian 
la conformación de grupos de trabajo y de investigación y fortalecen el trabajo colaborativo (Merchán et al., 2018).

4. En el aprendizaje de temas específicos, permiten la búsqueda y la selección de información especializada, lo que favorece el autoaprendizaje, la retroalimentación y el acceso a otras fuentes de información, que no solo refuerzan el conocimiento en temas específicos, sino que, además, facilitan el autoconocimiento (Limas et al., 2018; Merchán et al., 2018).

5. En la interacción y el debate sobre temas del curso, las clases se pueden complementar con actividades en las redes sociales mediante la creación de grupos cerrados (Educaweb, 2018) en donde se promueva la participación, el diálogo, la argumentación y la discusión sobre los temas del curso.

6. Como en las redes se publica y se comparte recursos y/o contenidos afines a los temas del curso, ellas permiten compartir noticias, documentos, imágenes, audios, videos, enlaces a páginas web, etc. (Viadeo, 2011), relacionados con los temas del curso, así como publicar información resultado del trabajo académico, al tiempo que se desarrollan competencias en el manejo de herramientas tecnológicas y digitales para el desarrollo profesional y laboral, de acuerdo con las necesidades actuales de la sociedad del conocimiento (Brenes, 2010; Laguna, 2013; Merchán et al., 2018).

7. En la investigación y elaboración de actividades, estas herramientas propician espacios de indagación y consulta acerca de los diferentes temas de los cursos, por lo que fortalecen habilidades y destrezas conceptuales que conllevan el aporte de ideas y reflexiones, mejoran la orientación durante la elaboración de las actividades y favorecen el aprendizaje (Limas et al., 2018).

8. Las redes sociales son fuente valiosa de consulta de contenidos y recursos y medio para intercambiar información que puede facilitar, ampliar y apoyar la elaboración de las actividades del curso (Limas et al., 2018; Florido, 2016).

9. Por dar acceso a cualquier hora, las redes sociales significan disponibilidad permanente de materiales y fuentes, adaptables al tiempo de los estudiantes (Brenes, 2010; Laguna, 2013).

El estudio no solo encontró ventajas, pues el uso de las redes sociles también tiene las siguientes desventajas como estrategia académica en la educación superior:

1. Dependencia de las redes sociales, que desborda tiempos y límites, debido a su uso inadecuado y/o sin control (Rial et al., 2014; Florido, 2016; Redem, 2017). Así se incurre en prácticas nocivas, por lo que es necesario concientizar al estudiantado acerca de su utilización como herramientas más de formación y de complemento de las demás, a las que no sustituye (Educaweb, 2018).

2. Distracción exagerada y exceso de información sobre diferentes temáticas, desventaja que, unida a la anterior, hace necesario promover el uso seguro de las redes sociales y establecer normas y límites (Redem, 2017), de manera que se genere una cultura de responsabilidad que extraiga el beneficio de estas herramientas y se minimicen sus riesgos (Marquès, 2008).

3. Dada la reducción del contacto físico entre compañeros y con los docentes, unida a los cambios de modalidad académica de la formación en bachillerto (presencial) a la formación universitaria (para el caso, el programa virtual), se pueden generar en los estudiantes cambios bruscos hacia nuevas modalidades de estudio hasta perder habilidades de socialización con los demás, y ello a pesar de que las redes sociales facilitan los canales y las oportunidades de establecer contacto entre compañeros y docentes vía chat, videollamada, mensajería, videoconferencia, etc. (Marquès, 2008). 
4. Los problemas de veracidad y confiabilidad en la información consultada se consideradan una desventaja, por estar disponible mucha información errónea y desactualizada, desde que cualquiera puede subir información. Por eso, los estudiantes deben fortalecer competencias encaminadas a seleccionar y hacer uso correcto de la información, así como a evaluar la calidad y la idoneidad de la misma (Marquès, 2008; Laguna, 2013).

5. Por la dificultad en el acceso a internet, algunos estudiantes pueden encontrar limitantes para acceder a las redes sociales, bien sea porque no cuentan con conexión o porque hay áreas geográficas en donde no llega la señal o el cubrimiento de internet es limitado. Por eso en las universidades se debe facilitar su acceso a equipos de cómputo con internet y/o conexión wifi de uso compartido, en horarios accesibles para ellos (Educaweb, 2018).

6. La mala administración del tiempo, que desplaza otras prioridades, sucede cuando de manera habitual se es incapaz de controlar el tiempo de conexión a las redes sociales y se posterga el desarrollo de otras actividades $y / u$ obligaciones (Marquès, 2008). Esta desventaja, junto con las dos primeras, exige capacitación en el uso adecuado de las redes sociales con miras a generar responsabilidad en su utilización. Por lo tanto, es necesario establecer pautas de buen uso de las redes sociales en lo que respecta al tiempo, el acceso y los límites de los estudiantes. Acá son claves el acompañamiento y la asesoría de los docentes -que deben planificar previamente las estrategias de acción, de acuerdo con el enfoque pedagógico que se implemente (Brenes, 2010)-, así como la capacitación de los estudiantes en la utilización y buenas prácticas en las redes sociales y la adecuada orientación en aspectos de seguridad informática (Castillo, 2013).
Por otra parte, este estudio pudo identificar que los estudiantes buscan opciones prácticas para su aprendizaje en el entorno universitario y asimilan con propiedad las herramientas de las TIC, en los entornos online, que facilitan el acceso a las redes sociales que les ofrece el siglo XXI (González y Muñoz, 2016; Maldonado et al., 2019).

Los resultados muestran que las redes sociales se deben incluir en los programas académicos como política institucional, por las ventajas señaladas para los procesos educativos, en beneficio de estudiantes y docentes. De igual forma, como herramienta pedagógica son primordiales, dadas las potencialidades que ofrecen (Brenes, 2010; González et al., 2016).

La educación actual no puede ignorar los cambios tecnológicos en torno a la comunicación y la nueva forma de creación, envío y recepción de la información, donde el profesor se ve obligado a replantear las estrategias y herramientas didácticas que el desarrollo tecnológico abre a los entornos educativos. Los estudiantes tienen las habilidades y capacidades para adaptarse rápidamente a los cambios tecnológicos y hacer uso de sus ventajas.

Las redes sociales se deben incluir en los programas académicos como política institucional, vistas sus bondades para la educación superior. En tal sentido, como herramienta pedagógica es primordial su incorporación, comprobadas las potencialidades que ofrecen en un entorno tecnológico mediado por las TIC, lo que conlleva que en los procesos de enseñanza-aprendizaje se incorporen al contexto universitario otras innovaciones de caracter académico.

En cuanto a las limitaciones del estudio, como los resultados y conclusiones toman como base la opinión y óptica del estudiantado, el estudio se puede enriquecer, si los resultados se analizan y se comparan con la perspectiva y la óptica de los docentes universitarios, a partir de sus experiencias en el quehacer educativo y el campo de formación. Por tanto, 
se recomienda integrar estudios con docentes atendiendo a la incorporación de las redes sociales en los procesos educativos, lo que permitirá ampliar y optimizar el análisis, al dimensionar los resultados desde las dos ópticas: estudiantado y docentes. Para investigaciones futuras, se recomienda asimismo realizar estudios en conjunto con otros centros educativos universitarios, a fin de fortalecer los resultados con su experiencia educativa y de formación. De igual manera, se sugiere incorporar nuevas variables de estudio: modalidad de estudio (presencial/ virtual) y nivel de formación (pregrado/posgrado).

\section{Referencias}

Ángel Uribe, I. C. y Patiño Lemos, M. R. (2018). Línea base de indicadores de apropiación de TIC en instituciones educativas. Educación y Educadores, 21(3), 435-457. https://doi.org/10.5294/edu.2018.21.3.4

Brenes Matarrita, O. L. (2010). Potencialidades de internet como herramienta pedagógica en la educación superior. Innovaciones Educativas, 11(16), 1-19. https://doi.org/10.22458/ie.v11116.549

Brito, J. G., Laaser, W. y Toloza, E. A. (2012). El uso de redes sociales por parte de las universidades a nivel institucional. Un estudio comparativo. Revista de Educación a Distancia, 1-38. https://www.um.es/ead/red/32/ laaser_et_al.pdf

Cabero Almenara, J., Barroso Osuna, J., Llorente Cejudo, M. d. C. y Yanes Cabrera, C. (2016). Redes sociales y Tecnologías de la Información y la Comunicación en Educación: aprendizaje colaborativo, diferencias de género, edad y preferencias. Revista de Educación a Distancia, 51, 1-23. http://dx.doi.org/10.6018/red/51/1

Cabrero, J. y Marín, V. (2014). Posibilidades educativas de las redes sociales y el trabajo en grupo. Percepciones de los alumnos universitarios. Comunicar, 42, 165-172. https://doi.org/10.3916/C42-2014-16

Cantillo Julio, C. J. y Moreno May, A. P. (2016). Uso y percepciones de las redes sociales en el proceso de enseñanaza-aprendizaje en el programa de Comunicación Social de la Universidad de Cartagena. [Proyecto de Grado], Programa Comunicación Social, Facultad de Ciencias Sociales y Educación, Universidad de Cartagena.

Castillo Ríos, R. A. (2013). Ventajas y desventajas del uso de las redes sociales en el estudio universitario de alumnos de antiguo y nuevo ingreso de la Universidad Francisco Gavidia (UFG) sede San Salvador. [Trabajo de cátedra en métodos y técnicas de investigación] Salvador.

Chacón Díaz, L. F.y Limas Suárez, S. J. (2019). Los cursos virtuales orientados por competencias: una mirada hacia la pertinencia e innovación educativa y tecnológica del siglo XXI. Revista Ibérica de Sistemas e Tecnologias de Informação, E2O, 113-125. https://search.proquest.com/openview/24835c788dbfaeo6c71df3c751073eaz/1. pdf?pq-origsite $=$ gscholar $\&$ cbl $=1006393$

Chávez Martínez, J. d. J. (enero-junio de 2014). Las redes sociales en la educación superior. Educación y Desarrollo Social, 8(1), 102-117. https://revistas.unimilitar.edu.co/index.php/reds/article/view/586 
Colina Colina, L. (2008). Las TIC en los procesos de enseñanza-aprendizaje en la educación a distancia. Laurus, 14(28), 295-314. https://www.redalyc.org/pdf/761/76111716015.pdf

Cornejo Álvarez, J. F. y Parra Encinas, K. L. (2016). Impacto de las redes sociales en los procesos de enseñanza aprendizaje de la educación superior pública. Boletín Redipe, 5(11), 1-22. https://revista.redipe.org/index. php/1/article/view/152

Educaweb (2018). Redes sociales: ventajas y desventajas para la formación. [Portal web]. https://www.educaweb. com/noticia/2018/04/16/redes-sociales-ventajas-desventajas-formacion-16408/

Escorcia Oyola, L. y Jaimes de Triviño, C. (2015). Tendencias de uso de las TIC en el contexto escolar a partir de las experiencias de los docentes. Educación y Educadores, 18(1), 137-152. https://doi.org/10.5294/edu.2015.18.1.8

Florido, M. (2016). Ventajas y desventajas de las redes sociales en la educación. Universidad Católica de Valencia. https://online.ucv.es/marketing/ventajas-y-desventajas-de-las-redes-sociales-en-la-educacion/

García Cué, J. L., Santizo Rincón, J. A. y Alonso García, C. M. (2009). Uso de las TIC de acuerdo a los estilos de aprendizaje de docentes y discentes. Revista Iberoamericana de Educación, 2(48), 1-14. https://rieoei.org/ historico/deloslectores/2308Cue.pdf

González Martínez, J., Lleixà Fortuño, M. y Espuny Vidal, C. (2016). Social networks and higher education: the attitudes of university students towards the educational use of social networks, back to test. Education in the Knowledge Society, 17(2), 21-38. https://revistas.usal.es/index.php/eks/article/view/eks20161722138

González, C. y Muñoz, L. (2016). Redes Sociales: su impacto en la Educación Superior: caso de estudio Universidad Tecnológica de Panamá. Campus Virtuales, 5(1), 84-90. http://uajournals.com/ojs/index.php/campusvirtuales/article/view/117

Hernández Infante, R. C. y Infante Miranda, M. E. (2017). La clase en la educación superior, forma organizativa esencial en el proceso de enseñanza-aprendizaje. Educación y Educadores, 20(1), 27-40. https://doi. org/10.5294/edu.2017.20.1.2

IAB Spain (2019). Estudio Anual Redes Sociales 2019. [Portal web]. https://iabspain.es/estudio/estudio-anual-deredes-sociales-2019/

Islas Torres, C. y Carranza Alcántar, M. d. (2011). Uso de las redes sociales como estrategias de aprendizaje. ¿Transformación educativa? Apertura, 3(2). http://www.udgvirtual.udg.mx/apertura/index.php/apertura/article/view/198/213

Laguna Segovia, M. I. (2013). Estudio sobre el uso del Internet y sus aplicaciones en el alumnado de último año de carrera de la Universidad de Alicante. [Tesis doctoral] Investigación educativa: Enseñanza y Aprendisaje, Facultad de Educación, Universidad de Alicante.

Limas Suárez, S. J. (2019). Los Objetos Virtuales de Aprendizaje - OVA en la Educación Superior como estrategia formativa en la era digital. Una experiencia a partir del curso Introducción a la Administración. En ATICA 
2019: Aplicaciones de Tecnologías de la Información y Comunicaciones Avanzadas (pp. 286-293). Universidad de Alcalá y Universidad Nacional de Córdoba. https://drive.google.com/file/d/1t5idBn-loHyqlvlVwukpnUKVIe-NLJGp/view

Limas Suárez, S. J. y Jaimes Reyes, A. M. (2017). Impacto de la educación virtual en los estudiantes del programa Administración Comercial y Financiera de la Universidad Pedagógica y Tecnológica de Colombia. En Aplicación del saber: casos y experiencias (vol. 3, pp. 3424-3430). Academia Journals.

Limas Suárez, S. J., Jaimes Reyes, A. M. y Salazar Araujo, E. J. (2018). La educación virtual en los programas de Educación Superior en el área de Administración, Colombia. En Compendio de investigación (pp. 1337-1343). Academia Journals.

López Neira, L. R. (2017). Indagación en la relación aprendizaje-tecnologías digitales. Educación y Educadores, 20(1), 91-105. https://doi.org/10.5294/edu.2017.20.1.5

Maldonado Berea, G. A., García González, J. y Sampedro-Requena, B. E. (2019). El efecto de las TIC y redes sociales en estudiantes universitarios. RIED. Revista Iberoamericana de Educación a Distancia, 22(2), 153-176. http:// dx.doi.org/10.5944/ried.22.2.23178

Marín Díaz, V. y Cabero Almenara, J. (2019). Las redes sociales en educación: desde la innovación a la investigación educativa. RIED. Revista Iberoamericana de Educación a Distancia, 22(2), 25-33. http://revistas.uned.es/ index.php/ried/article/view/24248/19893

Marquès Graells, P. (2008). Los riesgos de internet. Consejos para su uso seguro. Habilidades necesarias para utilizar internet. Departamento de Pedagogía Aplicada, Facultad de Educación, Universidad Autónoma de Barcelona. https://ddd.uab.cat/pub/dim/16993748n2/16993748n2a4.pdf

Martínez Rámila, K. P. y Ramírez Martinell, A. (2016). Redes sociales en educación superior: transformaciones tecnológicas, de socialización y de colaboración entre estudiantado universitario. Ensayos Pedagógicos (edición especial), 93-111. https://www.revistas.una.ac.cr/index.php/ensayospedagogicos/article/ view/9342/11072

Martínez, M. Y. (2014). Redes sociales y TIC en educación superior. Historia y Comunicación Social, 19(especial), 63-71. https://revistas.ucm.es/index.php/HICS/article/download/45108/42477/

Merchán Carreño, E. J., Mero Suárez, K. V. y Mero Suárez, C. R. (2018). Uso didáctico de las redes sociales en la educación superior. Sinapsis, 2(13). https://doi.org/10.37117/s.v2i13.158

Muñoz Prieto, M. d. M., Fragueiro Barreiro, M. S. y Ayuso Manso, M. J. (2013). La importancia de las redes sociales en el ámbito educativo. Escuela Abierta, 16, 91-104. https://dialnet.unirioja.es/descarga/articulo/4425349.pdf

Newman, N., Fletcher, R., Kalogeropoulos, A. y Kleis Nielsen, R. (2019). Digital News Report 2019. Universidad de Oxford, Reuters Institute for the Study of Journalism.

Prato, L. y Villoria, L. (2010). Web 2.o: Redes sociales. Universidad Nacional de Villa María. 
Redem (2017). Educación 3.0: Usos de las redes sociales en el aula. Ventajas y desventajas. Redem: Red Educativa Mundial. [Portal web]. https://www.redem.org/educacion-3-o-usos-de-las-redes-sociales-en-el-aula-ventajas-y-desventajas/

Rial, A., Gómez, P., Braña, T. y Varela, J. (2014). Actitudes, percepciones y uso de Internet y las redes sociales entre los adolescentes de la comunidad gallega (España). Anales de Psicología, 30(2), 642-655. http://dx.doi. org/10.6018/analesps.30.2.159111

Rojas Castro, P. (2016). Learning analytics: una revisión de la literatura. Educación y Educadores, 20(1), 106-128. https://doi.org/10.5294/edu.2017.20.1.6

Romero Delgado, C. (2012). Diseño y desarrollo de un ambiente de aprendizaje al estilo EDUPUNK: La historia del diseño con recursos de la web 2.o. Primer Congreso Internacional de Educación: construyendo inéditos viables. Universidad Autónoma de Chihuahua. http://cie.uach.mx/cd/docs/area_o2/a2p6.pdf

Sáenz López, J. M. (2010). Utilización de las TIC en el proceso de enseñanza-aprendizaje, valorando la incidencia real de las tecnologías en la práctica docente. Docencia e Investigación, 20, 183-204. http://e-spacio.uned. es/fez/eserv/bibliuned:425-Jmsaez-1085/utilizacion_tic.pdf

Shang, S., Li, E. Y., Wu, Y.-L. y Hou, O. C. (2011). Understanding web 2.0 service models: a knowledge-creating perspective. Information \& Management, 48, 178-184. https://doi.org/10.1016/j.im.2011.01.005

Silva Robles, C., Jiménez Marín, G. y Zambrano, R. E. (2012). De la sociedad de la información a la sociedad digital. Web 2.0 y redes sociales en el panorama mediático actual. Revista f@ro, 1-14. https://dialnet.unirioja.es/ descarga/articulo/4160011.pdf

Valenzuela Argüelles, R. (2013). Las redes sociales y su aplicación en la educación. Revista Digital Universitaria, 14(4). http://www.revista.unam.mx/vol.14/num4/art36/art36.pdf

We Are Social (2019). Global Digital Report 2019. https://wearesocial.com/global-digital-report-2019 